\title{
Digital Humanities and the Interdisciplinary Database: Confronting the Complexity of Chinese Religious Architecture in the Academic Marketplace
}

As most of us now realize, the use of digital tools to engage in computational, interdisciplinary research in the humanities holds the potential to transform our understanding of the human condition, past and present. However, in order to take advantage of the power of digital tools, data previously constructed for publication in book form must be reconceptualized for both analog and digital consumption. The success of a young digital humanities scholar, one who is required to produce peer-reviewed publications for tenure and promotion, is partially dependent on clarifying the front-end time commitment of tool development and data entry necessary for the development of current digital projects. The dynamic, trial-and-error nature of the field can make even this task difficult. However, as more and more tools are developed for the study of the material culture of religion, selecting the appropriate items and reorganizing them for customized applications promises new ways of researching and understanding the historical past. The collaborative culture of digital humanities increasingly allows for the development of more efficient ways to produce tangible results so that younger scholars can succeed in both project and career.

This essay is a documentation of my experience developing Architectura Sinica, a digital humanities project designed to facilitate research in medieval Chinese temple culture. I will begin by providing a background to the project scope and associated image archives to give a sense of the nature of art historical teaching and research tools, past and present. I will then discuss my efforts to actualize the project through the semantic web and through XQuery languages and technologies. Finally, I discuss my current application and some of the challenges I have confronted with text and image management. I hope this essay will help introduce scholars interested in beginning a digital material culture project in the academic environment to a few of the pathways forward, as well as some of the roadblocks, in computational humanities. 


\section{Assessing the Scope of the Project}

Architectura Sinica started as a biproduct of fundamental art historical research on regional style in traditional Chinese timber-frame architecture from the Middle Period (roughly the tenth through the twelfth centuries). In my dataset, the individual buildings and sites that contained them are all dedicated to divinity worship. Most of the sites are Buddhist, but temple buildings and complexes dedicated to divinities associated with monastic Daoism, as well as Confucianism, state religion, and local cults, are included. Early on it was clear that the results of this research could be of interest to an interdisciplinary audience including art/architectural historians interested in Chinese art and global building technology, as well as historians of religion interested in material culture of ritual praxis in Asia.

My original concept seemed very simple. I wanted to create a research tool that would allow me to identify specific stylistic and structural details of these historic buildings, search by those architectural features, and map the output to determine whether stylistic differences were related more to regional distinctions or trends in an empire-wide style that changed gradually over time. In order to problematize the question of dynastic style, I chose to focus on a group of historic buildings from a particular time period rather than a dynasty. I selected the period from $900-1200$ because within this date range we have sufficient extant historical remains in different areas of China to investigate variations in structure and style over space as well as time. Therefore, it seemed that the use of GPS technologies to both document the location of individual sites, buildings, and other artifacts (including sculpture and stele inscriptions) and dynamically map development over time would be a fruitful research endeavor.

To build the database it was necessary first to determine the individual component parts of the sites within the study. Traditional Chinese architecture is routinely organized into large building complexes (which I refer to as "sites"), and historic buildings within the complex are usually identified by both the name of the site as well as the name of the building itself. For example, we might have a Great Buddha Hall (individual historic building) within Dayun Cloister (larger site), which would usually be called the Dayun Cloister Great Buddha Hall. The architectural features which are the focus of my research are associated with individual buildings. Other artifacts, including sculpture, paintings, and stele with inscriptions are also contained within a site. The larger site is typically older, sometimes substantially so, than the extant buildings and other artifacts contained within it. Thus, I would need to have a separate entry or web page con- 
taining information on the site that was distinct from the web page containing information on the building. Because the terminology for the sites, buildings, and individual architectural features is unfamiliar to non-specialist readers in Chinese as well as in English or other languages we needed to have a dictionary function of some kind as a separate portion of the website.

Trained in the days of text tables and analog photography, the biggest surprise in embarking on this project was realizing I needed to fully rethink my documentation methods for the sake of computer consumption and output. By 2007 I had already completed a significant portion of the research on individual buildings, with field notes and photographs for the forty-one most important of the one hundred twenty-three complexes believed to hold authentic buildings from the period. I received an NEH grant in 2008 to develop my regional style project with the potential for building an open-access web-based research tool as part of the larger proposal. Improvements in commercial mapping systems, such as Google Earth, revealed the immediate potential of the graphic display of my GIS data, and encouraged me to consider developing the digital archive of photographs and a mapping website as a first step in my regional style project. Yet, although the tables and spreadsheets I created for my own use in books and articles were appropriate for print publication and remained good research tools, they were not appropriate for computerized data manipulation. I needed to reformat my textual data completely.

Furthermore, by 2007 technologies for producing raw images of buildings, a data source as important as textual documentation for an architectural historian, had undergone their own digital revolution and technologies for storing and manipulating those images were, and continue to be, rapidly changing. Images of the buildings I studied from 1997-2002 had all been produced as analog slides and photographs. As digital cameras improved and became more affordable in the early 2000s, the shift to born-digital images created new challenges for data storage and documentation.

Transformations in the production of images for research purposes were paralleled by the rapid shift from analog to digital in art and art history instruction, a development that directly impacted the infrastructure for research and study of cultural heritage objects. This was not the first time technological developments dramatically transformed the means by which we come to learn about the physical world around us. Photography itself, of course, began that process, and with it a field developed around the preservation and cataloging of photographic representations of cultural heritage. Within ten years of the development of photograph, plate glass was used for positive photographic transparencies, as well as negatives which would then be projected onto photographic paper. The positive transparencies could be projected onto a wall at a magnified scale with the 
“magic lantern," a technology for displaying images used as early as 1665 . These lantern slides were quickly used for research on subjects as diverse as historic architecture and medical research. ${ }^{1}$ Lantern slides were used for instruction in the art history classroom beginning in the late nineteenth century, and continuing at least through the 1960s, by which time 2x2" glass slides and then $35 \mathrm{~mm}$ slides replaced the larger-format lantern slides, and the lantern slides were relegated to the archive. ${ }^{2}$

The digital revolution of the early twenty-first century had a similar impact on the study and teaching of material culture. As advancements were made in scanning, digital photography, projection, and file storage, teaching with digital reproductions, which could be saved to a personal computer rather than filed in a slide library, was an obvious choice. The convenience of digital imaging was made a necessity by 2004, when Kodak discontinued the production of slide projectors. ${ }^{3}$ Slide libraries were quickly transformed into visual resources centers for the support of slide scanning, digital image archiving, and training in the digital image presentation software now indispensable for day-to-day classroom instruction. Vanderbilt was on the cutting edge in the use of this technology, and faculty were already using digital images in virtual slide trays for classroom instruction in the 2001-2002 academic year. ${ }^{4}$ With a move to a new facility in 2009, all art history course presentations were moved to digital platforms (mostly PowerPoint and Keynote) and the slide collection at Vanderbilt University was, like the earlier lantern slides, placed into off-campus storage. Thus, during this same period my own system for presentation and publication of images,

1 Simon H. Gage, “The Introduction of Photographic Transparencies as Lantern Slides,” Journal of the Royal Society of Arts 59.3036 (1911), 255-257.

2 Annemarie van Roessel, "Through a Glass, Brightly: Re-viewing a Lost Architectural and Pedagogical Landscape Through Historic Lantern Slides," Art Documentation: Journal of the Art Libraries Society of North America, 22.1 (2003): 6. Developments in color photography in midcentury helped to reduce costs and facilitated the shift in format, see Carla Conrad Freeman, "Visualizing Art: An Overview of the Visual Resources Profession in the United States," Art Documentation: Journal of the Art Libraries Society of North America, 7.1 (1988): 6.

3 Ching-Jung Chen, "Analog to Digital: Conversion of the Image Libraries at the City College of New York," Art Documentation: Journal of the Art Libraries Society of North America, 28.1 (2009), 36. https://web.archive.org/web/20050206010328/http://slideprojector.kodak.com/ (accessed October 25, 2018).

4 Marshall Breeding, “A Web-Based Image Access System for Classroom Presentation in Art History,” Coalition for Networked Information Project Briefings, April 17, 2002. https://www.cni.org/ topics/digital-curation/a-web-based-image-access-system-for-classroom-presentation-in-art-his tory (accessed January 18, 2019). 
the primary source data on which my argument hinged, needed a full overhaul as well.

I realized that I needed to restructure my data for two purposes: efficiency of cataloging numerous images of buildings and artifacts tied to a temple site, for which a relational database seemed appropriate, and managing research notes and sources on a site-all textual data-in a format that would allow for the greater understanding of regional architectural style and sharing of that information digitally. These two purposes had multiple options that I was cautioned to consider before setting up the system's architecture overall. In the end I decided that the cataloging of textual information might be better served by one set of tools and building an archive of images of objects by another. Although differently organized, information on images and artifacts could be pulled together for display through a web portal. The promise of Linked Data and SPARQL (SPARQL Protocol and RDF Query Language) that was so compelling at the beginning of the project was retained as an area of future development as all of our data can be exported in RDF (Resource Description Framework)/XML as well as TEI (Text Encoding Initiative)/XML and GeoJSON. But a lot of experimenting was necessary before I came to those conclusions.

\section{Reconceptualizing Art Historical Data}

The reorganization of textual data began with a fresh look at tables and spreadsheets. My fieldwork starts with photo-documenting extant premodern temple complexes and taking field notes regarding geolocation, rough building dimensions, and description of critical architectural features. Beginning in 1997 I used simple tables within the word processing program Nisus, because of its ability to handle Chinese characters. I printed these to fill in on site, and then manually input the data into my computer after returning to an indoor environment. As the MS Word suite improved its ability to handle non-Latin scripts, I began using Excel spreadsheets and, beginning in 2007-2008, I printed these in booklet form for each fieldtrip. These were necessarily compact for their use in the field, so all of the different elements I wanted to ensure were included in my fieldwork were configured to be able to print onto a single $8.5 \times 11$ " page (Fig 1).

After deciding I wanted to engage in a computational project, I learned my spreadsheets were not machine readable. That is, while these spreadsheets were "digitized," they were not easily consumed by database programs and thus what 


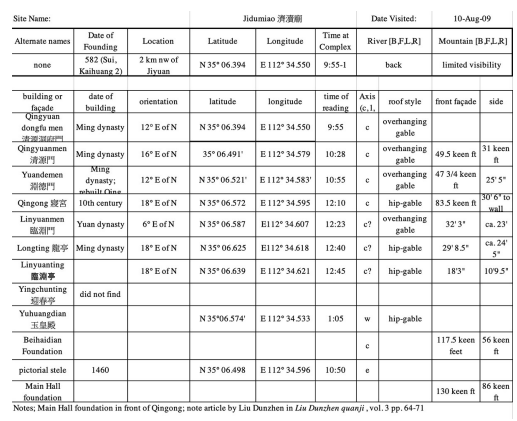

Figure 1: Example of site spreadsheet designed for use in the field.

I was doing was not actually "digital humanities," strictly defined..$^{5}$ After consulting first with the Vanderbilt campus GIS specialist (Jacob Thornton) and later with scholarly communications specialists (Cliff Anderson and Steve Baskauf), I learned that software developed for humanities computing, including GIS and XQuery, is often designed to consume CSV (comma-separated values) documents. Yet, while tables pose no problem for organizing my humanistic data, organizing by comma-separated values was extremely difficult for me to edit or otherwise control.

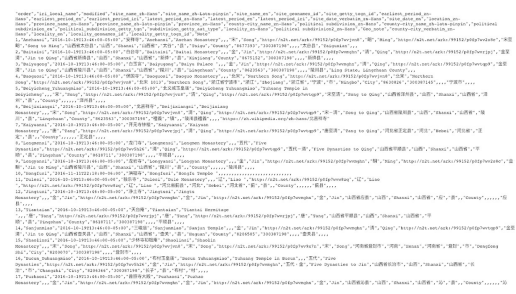

Figure 2: Screenshot of sites spreadsheet viewed in "raw" format on GitHub.

Although spreadsheets in CSV format are considered to be more user-friendly than a document in simple comma-separated values viewed without visible cells or "raw," (Fig. 2), they are still extremely cumbersome to fill and edit in analog format and consequently less functional in the field (Fig. 3).

My first experiment was to generate a map of the individual building sites in my dataset. In order to manipulate my more complex metadata, I would need to reorganize it into a simple, unformatted, two-dimensional spreadsheet. For the

5 For more on what the Digital Humanities is and is not, see Burdick, Anne, et al. "Questions and Answers: Digital Humanities Fundamentals.” In Digital Humanities, edited by Anne Burdick, Johanna Drucker, Peter Lunenfeld, Todd Presner, and Jeffrey Schnapp (Cambridge, Mass., and London: The MIT Press, 2012), 122. 


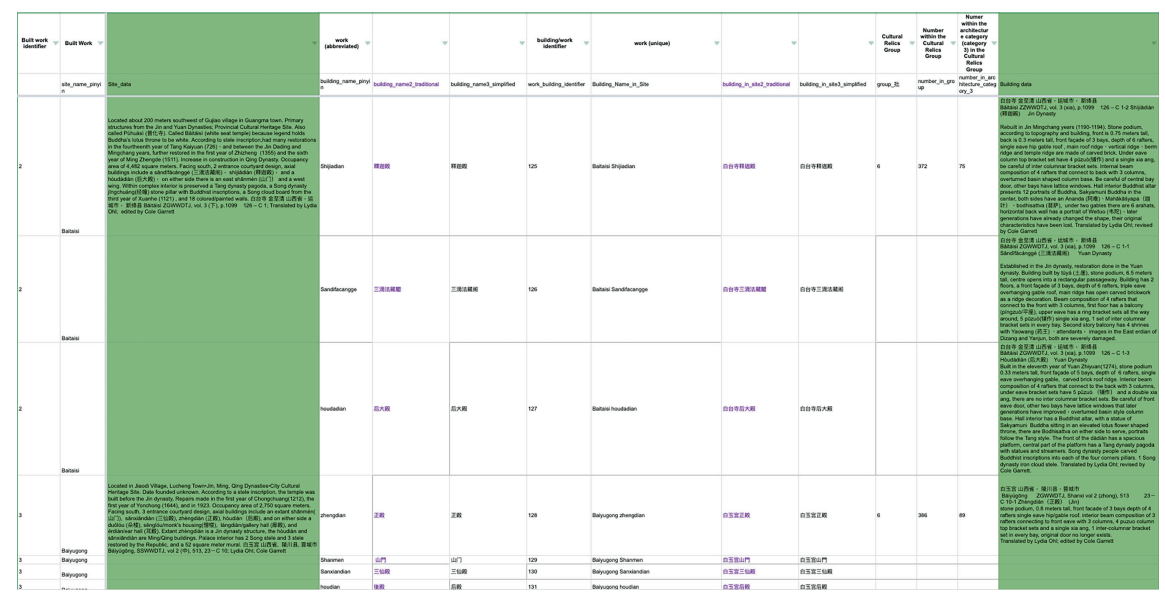

Figure 3: Screenshot of Google Sheets version of combined site/buildings spreadsheet.

map I created a flat spreadsheet with rows with a column for the name of each site, its latitude, and its longitude. I also converted the latitude and longitude taken from my hand-held GPS from analog to digital coordinates in order to be able to take advantage of mapping software like ArcGIS. Of course, once in CSV format with digital coordinates, the same spreadsheet could be used to create as many maps as I wanted, and I could write a program (or use a pre-existing one) to select any number of sites based on particular search criteria. I was reassured by one colleague that CSV is still the most stable document type, so this was not the worst way to ultimately store data and therefore not a waste of precious research time-which, to be honest, was how it felt. Still, this appeared to be a relatively straightforward task. The subsequent deconstruction and reconstruction of the rest of my data followed two paths, both of which are preserved in the Architectura Sinica website today.

\section{Coding Nomothetic vs. Idiographic Data}

The types of data organization I needed to produce my ideal web output brought into focus methodological differences between digital humanists and scientists, much like Neo-Kantian dichotomies between nomothetic and idiographic approaches to knowledge. With generalizable nomothetic data, the binary computational model is easily adaptable. Geolocation of the individual buildings, for example, went very quickly from spreadsheet to output in maps, and was also readily adaptable to RDF formatting for sharing through LOD (Linked Open 
Data). For more idiographic data, especially where we wanted to build in the potential to account for multiple interpretations, XML languages provide greater potential to account for idiosyncrasies, a process more in keeping with the long tradition of textual interpretation. ${ }^{6}$ When I began my project, participants in the working groups addressing DH topics at Vanderbilt were willing to experiment with my data in two different systems. The first was based in the promise of sharing through LOD using RDF triples, the second was the rewriting of the code according to TEI guidelines in order to be able to better document the historical and, potentially, epigraphical elements associated with my building sites.

Our Semantic Web working group was the first to take on my dataset. Led by one of the group members, biologist Steve Baskauf, my data were used to test a piece of code, the "Guid-O-Matic," that he had developed from the need to generate global unique identifiers for his Bioimages database. Interested in bioinformatics, Baskauf wrote a light program to transform flat CSV spreadsheets of his data into RDF triples for consumption through a SPARQL endpoint. ${ }^{7}$ Having established HTTP URIs as unique identifiers for trees and other species in Bioimages, Baskauf was interested in updating the program, and I was interested to see if it might be applicable to the study of cultural heritage sites. Working with two other colleagues in the group, one from our central library (Yuh-Fen Benda) and the other from our visual resources center (William Sealy), we pulled together the essential searchable categories of objects into a spreadsheet, and developed a data model for the categories. Originally, we were hoping to be able to display the site in different languages and scripts, and so all of the text data (site names, building names, and architectural features) were described in English, traditional Chinese, and simplified Chinese.

My first spreadsheet, one which I could follow as a human, was still not really simple enough for computer consumption, and especially not for the parameters of Linked Data (Fig. 3). It was an all-encompassing single document that reflected complex relationships between an individual historic building, a larger historic building complex (or cultural heritage site), and a location with numeric (latitude/longitude) and text (modern country, province, town) reference points. For XQuery queries, searchable categories need to be considered individually. In one work-group session we discussed the relationships between the various com-

\footnotetext{
6 Charlotte Roueché, “Why Do We Mark Up Texts?” In Collaborative Research in the Digital Humanities, edited by Willard McCarty and Marilyn Deegan, 118-123 (Farnham, Surrey, England: Ashgate, 2011).

7 For more on Steve Baskauf's development of the initial Traditional Chinese Architecture Digital Research Tool, see the detailed discussion on his blog: http://baskauf.blogspot.com/2016/10/ guid-o-matic-goes-to-china.html (accessed Jan. 2019).
} 
ponents of my data to establish how we would want them to be able to relate to each other in a search. Once those relationships were mapped out (Fig. 4), Baskauf used his modified Guid-O-Matic code, GOM2, to create a web application for display. Because of the difference in the dating of sites and buildings, we needed separate spreadsheets for, and eventually also gave separate URIs to, each of the sites and each of the historic buildings (or artifacts) I had documented within the sites.

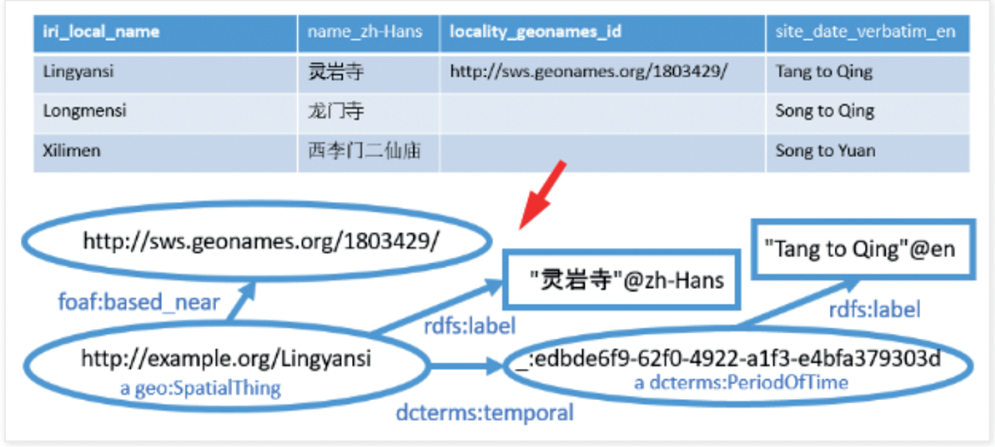

Figure 4: Bubble graph diagram and spreadsheet showing organization according to the Resource Description Framework (RDF) Source: http://baskauf.blogspot.com/2016/10/guid-omatic-goes-to-china.html.

The promise of the Semantic Web is interoperability, and Baskauf's goal for our group was to test the possibilities of XQuery and generate RDF-formatted LOD for consumption through SPARQL queries. To that end, he posted the code on GitHub for open download and sought out external sources of LOD to import into the application whenever possible. GitHub may be openly available, but it is not user friendly for the neophyte. And although I created a GitHub account and currently happily use GitHub to edit code, its core function, cloning code for individual experimentation, remains beyond my comfort-zone. Thus, while I was involved in some of the editing, at the time I depended on the developer in our visual resources center to manage our installation of Baskauf's code.

One of the primary goals of the Semantic Web working group was to experiment with different sources of Linked Open Data. These included GeoNames (seen in Fig. 4), the Getty Thesaurus of Geographic Names (TGN), and, potentially definitions of architectural terms that could be imported from the Getty Art and Architectural Thesaurus (AAT). Baskauf was also able to import research images by linking to my Flickr Pro account, where I store my born-digital images, and mapping data through Google Earth. As an experiment, this was very excit- 
ing. He was able to quickly generate a search engine that would display satellite and map images of sites, associate them with nearby locations using GeoNames, and import PRC-generated county and province names from the TGN for location display.

I was a bit uncomfortable employing these external sources for a stable research website, however, and I still strongly question the dependability of web sources for geographic information in China. For example, although Baskauf chose 1803429 as the location for a village/town of Lingyansi, that is only one of six entries with that name in GeoNames. Even the TGN poses a challenge for consistency because of the use of simplified characters for all of the Chinese place names. While the standard character set for the People's Republic of China is the simplified format, we would need to run a conversion program in order to display those names in a fully traditional Chinese character version of the site. Furthermore, because my subject area is still in an early stage of development, there are no reliable external authorities for much of my data. The Getty AAT, for example, did not contain terminology specific to historic Chinese buildings beyond one or two words. Indeed, I began to think that my dataset was not fully reducible to a few generalizable principles, and might not ever be. In order to account for the vagaries of information about the historic past, I turned to a different database model, one that was being developed by Dave Michelson on Syriac culture. For that I needed to have a full rewrite of the site, which I renamed Architectura Sinica.

Truth be told, the transition from the Traditional Chinese Architecture Digital Research Tool to Architectura Sinica resulted as much from the desire to collaborate with Vanderbilt colleagues to develop a portable cultural heritage application as it did from frustration with the current limitations of RDF and SPARQL queries. Still, I needed to be able to document my sources for seemingly objective pieces of information, such as the beginning and end dates I chose for Chinese dynasties in my general date information. I also needed to clearly document multiple sources of interpretation and disagreement within the blocks of text describing the sites, buildings, and other artifacts. Michelson and the team that developed the "Srophe app" for the Syriac Reference Portal had resolved many of these issues when developing their website. As stated on their site, the goals of their project were to compile sources on the study of Syriac culture and facilitate wide distribution of information and tools to further the study of the subject. ${ }^{8}$ It was written in the XML application eXist, using TEI guidelines. Like Baskauf's projects, information sharing is fundamental to their approach. The code can

8 http://syriaca.org/about-srophe.html (accessed January, 2019). 
be forked from GitHub for individual development and publications of Syriaca.org are openly available under Creative Commons licensing. Although their focus was on textual data rather than physical artifacts, they were also concerned with the physicality of their material and mapping is a big part of their project.

Michelson had already worked with a colleague in our Anthropology department, Steve Wernke, to adapt the code to his research in the Andes, and it seemed fruitful to try to customize their codebase for my dataset. ${ }^{9}$ I hired an independent developer, Winona Salesky, who had worked with Michelson and Wernke on their sites, to adapt the code for Architectura Sinica. Still a work in progress, during the 2018-2019 academic year, we hired a post-doctoral scholar to help regularize the code for Architectura Sinica and the Srophe app overall so that it conforms more closely to TEI guidelines. We are also fully documenting individual aspects so that the app might be customizable beyond the Vanderbilt campus. During the transition process I chose to make a number of simplifications based on my experience building the initial spreadsheets for TCADRT. The most significant of these was in the choice of display languages. Producing parallel sites in English, traditional Chinese, and simplified Chinese proved to be too cumbersome to edit effectively without a large team of bilingual specialists. I decided it would be better if the whole site were bilingual in English and Chinese. I also chose to use traditional characters rather than simplified. This would allow for inclusion of epigraphic information in the future, which is all written in traditional characters, and simplify editing. Yet, because we aspire to have a bilingual site, rather than a site displayed in different script sets (Roman, traditional Chinese, and simplified Chinese), I still feel comfortable using simplified characters for TGN imported geographic names. In this case it can be seen as less of a political position and more of a pragmatic one, as this is how maps generated in the target country would represent themselves.

The second change was to expand the architectural features section of the original site. My original dataset included only those architecture features I had deemed most critical to documenting stylistic change across different regions during the 900-1200 timeframe of my initial project. At first I imagined that we would be able to import other technical terms less critical to my study, such as the different building and site types common to Chinese religious architecture, to definitions in the Getty AAT using LOD through a SPARQL query.

9 Steve Wernke's site, developed with Jeremy Mumford, is the Linked Open Gazetteer of the Andean Region (LOGAR), see: http://www.logarandes.org/geo/about.html (accessed January, 2019). 
Yet, because the Getty AAT did not have material yet on Traditional Chinese Architecture, a number of my colleagues working in Chinese architecture and I thought it might be useful to research and define important terms for submission to the AAT. The first meeting of the ATTCAT (Annotation and Translation of Traditional Chinese Architectural Terminology) Project took place on June 21-26, 2018. We are slated to publish the results of our research on Architectura Sinica as well as through the Getty AAT, thus the results of this project, too, are designed for consumption through Linked Open Data. For the sake of community, and interoperability, I feel good about adopting TEI guidelines as we prepare individual entries for publication on Architectura Sinica. The Pictorial Dictionary of Chinese Architectural Terminology will adhere to the highest academic standards in the humanities, including detailed bibliographic references and a Works Cited page so that scholars using the site will always be able to trace the source of our information. Additionally, we are working to include drawings and photographic examples of idiosyncratic elements. Determining the best method of storing high quality image files is our next big challenge.

\section{For the Future: Images of Artifacts}

Although a core mission of the Traditional Chinese Architecture Digital Research Tool and the later Architectura Sinica was to make available images of extant tenth through twelfth century buildings in China, I have yet to determine the best means to archive and curate those images for internet consumption. As I mentioned above, Baskauf's initial code pulled images from Flickr to populate the relevant pages of my site, a system we are still using. But this commercial service was not my preferred image repository, precisely because it is subject to the vagaries of the marketplace. Below I briefly outline my experience building (or, more precisely not building) an interoperable image archive housed at the university. I also describe potential technologies we might adopt in the future.

Having been involved in the transition from analog to digital in our own Visual Resources Center, I had originally planned to archive and catalog images using DIMLI (Digital Media Management Library), a relational database our VRC staff developed in the wake of the 2008 financial crisis. Caught in the whirlwind of the transformation from analog to digital in the early 2000s, the Visual Resources Center at Vanderbilt experimented with a number of different archiving and image-delivery systems. As mentioned above, our first in-house system used during the 2001-2002 academic year replicated the slide tray exactly. The digital slide tray files were projected individually in sequence and required two 
digital projectors in order to project two images for comparison. Yet, the division of labor in the traditional slide library reflected a division between well-developed methods of cataloging and archiving physical slides and the technology used to produce them. Digital images seemingly brought them together: catalogers and digital photographers were both using computers. Yet the specialized training and intellectual inclination of digital photographers and slide curators were usually different, and unlike with slides, the image delivery system was more closely tied to the tools for cataloging than the tools for projection. Quality control fell first to those responsible for cataloging images, with image quality as a secondary concern because of the costs of high-resolution image storage and projection. Early iterations of endeavors to share digitized images, particularly through the Mellon-funded Artstor, fell short in both image quality and their digital image viewer, making the shift to digital more challenging for scholars who needed high quality reproductions for their research and teaching. ${ }^{10}$ Thus, in order to maintain a high level of instruction using the digital projector, we still needed (and still do need) to have means to produce and archive images unique to our teaching at the institutional level. We turned to MDID (Madison Digital Image Database), an open-source image repository and delivery tool for art history teaching developed at James Madison University. Although the image viewers in both Artstor and MDID were cumbersome to use, by this time the power of images in business presentations had fueled the commercial development of presentation software such as PowerPoint and Keynote and faculty quickly adopted them for everyday teaching as well as public lectures and conference presentations. Yet, funding cuts due to the 2008 financial crisis undermined development of MDID and we had to look elsewhere for our cataloging tools.

Concerns that MDID would fold due to lack of funding, coupled with a general disappointment in the quality of Artstor's Shared Shelf cataloging tool and archived images overall, inspired our VRC staff to develop their own cataloging and image retrieval tool. Artstor and MDID used flat cataloging systems, which were cumbersome to use for image cataloging. Development of our own inhouse cataloging tool, DIMLI, emerged from a desire to use a relational database for our image archive to enhance speed in cataloging. It also allowed for delivery of PowerPoint-sized images to faculty for classroom and conference presentation. However, institutional backing for this project was shaky, and retaining key staff as they enhanced their own technological profiles proved to be impossible. In the interim, Artstor expanded their tool for customized university ar-

10 A brief history of Artstor, and its new shared digital media platform, JSTOR Forum, can be found at http://www.artstor.org/mission. 
chives, originally Artstor Shared Shelf, to create JSTOR Forum, to which our teaching archive at Vanderbilt is currently being transferred. This tool can now support a relational database structure and remains a possibility for archiving my personal collection. Yet, even as I write, new image-sharing protocols are being developed for more sophisticated access to high-resolution images, including image annotation. A consortium of image repositories and research libraries is working to produce the International Image Interoperability Framework (IIIF). This system allows for remote image file viewing whereby the user can magnify a portion of an image for their research without downloading a large file to their desktop (or other) repository, among other functions. ${ }^{11}$ Major image repositories, including Artstor, are also working to be IIIF compliant, which bodes well for increased image sharing and manipulation in the future. In the interim, Flickr is a relatively inexpensive option for simple cataloging and image retrieval. As long as the company (or its current parent, SmugMug) remains viable, my database can continue to incorporate images.

\section{Conclusion: Lessons Acknowledged, If Not Yet Learned}

Developing Architectura Sinica has provided me an opportunity to reconceive the nature of the visual data I employ in my research. This digital turn allows for a more integrated research project with the potential for a much wider reach as text and material sources on sacred sites can be analyzed together using similar tools of organization. I feel confident it will suit my immediate research needs, and I hope that its availability on the web will expand interest in and an understanding of traditional Chinese architecture. As we expand the Srophe app for a larger audience, we have to acknowledge that GitHub and XQuery require a large time commitment, perhaps too large for most non-technical people to use efficiently. One way in which we are currently addressing this concern is by developing a fillable web form for individual entries that would allow data input with no knowledge of XML languages. Ideally it will be designed so that an undergraduate working in a lab can complete entry drafts with minimal supervision and editing. I have many colleagues who would take on more digital projects if similar tools were developed in order to maximize their humanities training

11 For more details on the benefits of IIIF, see https://iiif.io/community/faq/\#what-is-iiif (retrieved January, 2019). 
and minimize the need for coding skills. One is forced to ask, is facility with $\mathrm{XML}$, or RDF, a necessary qualification for the digital humanist?

As material culture becomes accessible to scholars of religion and other areas of the humanities, there will be even greater demand for a means by which to treat text as artifact and artifact as text. Increased accessibility of image and map data provides an opportunity for us to become more familiar with the complexity of the pre-modern past. IIIF encoding will greatly enhance the usability of high-quality digital reproductions, and has the potential to transform their use in the research through image annotation.

The application of digital tools for the study of religious sites will come into its own when university DH labs across the country are developed enough to include technical staff expert in industry standards for rapid digital archiving and data retrieval. This is already in place in many institutions, and will be greatly facilitated by the development of customizable open-source tools. Modification of commercial and social networking applications may be able to fast-track results, but within the university, an institution unique in its ability to generate and preserve scholarship of the highest level for the benefit of future generations, we should work to develop robust systems for high-level research independent of the fluctuations and fads of the marketplace. 
